Erratum

Gastroenterologe $2020 \cdot 15: 520$

https://doi.org/10.1007/s11377-020-00485-0

Online publiziert: 28. Oktober 2020

(c) Springer Medizin Verlag $\mathrm{GmbH}$, ein Teil von Springer Nature 2020

M.-W. Welker' - A. A. Schnitzbauer ${ }^{2}$

${ }^{1}$ Medizinische Klinik 1, Universitätsklinikum Frankfurt, Frankfurt am Main, Deutschland ${ }^{2}$ Klinik für Allgemein- und Viszeralchirurgie, Universitätsklinikum Frankfurt, Frankfurt am Main, Deutschland

\title{
Erratum zu: Aktuelle Aspekte der Immunsuppression nach Transplantation solider Organe
}

Erratum zu:

Gastroenterologe 2019

https://doi.org/10.1007/s11377-019-03

$57-y$

In $\bullet$ Tab. 1 des Originalbeitrags ist bei der Auflistung der zugelassenen Immunsuppressiva ein Fehler unterlaufen. Everolimus ist im Rahmen einer Erhaltungsimmunsuppression nach einer Lungentransplantation nicht zugelassen. Tacrolimus ist zur Immunsuppression nach Herztransplantation zugelassen. Bitte beachten Sie die korrigierte Version der Tabelle (geänderte Angaben in fettkursiv). Die Autoren bitten diesen Fehler zu entschuldigen und die korrekte Darstellung zu berücksichtigen.

\section{Korrespondenzadresse}

\section{M.-W. Welker}

Medizinische Klinik 1, Universitätsklinikum Frankfurt

Theodor-Stern-Kai 7, 60590 Frankfurt am Main, Deutschland

welker@med.uni-frankfurt.de

Tab. 1 Zugelassene Immunsuppressiva zur Rejektionsprophylaxe im Rahmen einer Erhaltungsimmunsuppression nach Herz-, Leber, Lungen, Niereund Pankreastransplantation

\begin{tabular}{|c|c|c|c|c|c|c|c|}
\hline & \multicolumn{2}{|c|}{ Antimetaboliten $^{\mathrm{a}}$} & \multicolumn{2}{|c|}{ Kalzineurininhibitoren ${ }^{\mathrm{a}}$} & \multicolumn{2}{|c|}{$\begin{array}{l}\text { Mechanistic-target-of- } \\
\text { rapamycine(mTOR)-Inhibitoren }{ }^{a}\end{array}$} & \multirow{2}{*}{$\begin{array}{l}\text { Steroide }^{\mathrm{a}} \\
\text { Z. B. Prednisolon }\end{array}$} \\
\hline & Azathioprin & $\begin{array}{l}\text { Mycophenolat- } \\
\text { Mofetil }\end{array}$ & Cyclosporin A & Tacrolimus & Everolimus & Sirolimus & \\
\hline Herz & Ja & Ja & Ja & $J a$ & $\mathrm{Ja}$ & Nein & Nein \\
\hline Leber & Ja & $\mathrm{Ja}$ & Ja & Ja & $\mathrm{Ja}$ & Nein & Nein \\
\hline Lunge & $\mathrm{Ja}$ & Nein & Ja & Nein & Nein & Nein & Nein \\
\hline Niere & $\mathrm{Ja}$ & $\mathrm{Ja}$ & Ja & $\mathrm{Ja}$ & $\mathrm{Ja}$ & $\mathrm{Ja}$ & Nein \\
\hline Pankreas & $\mathrm{Ja}$ & Nein & Ja & Nein & Nein & Nein & Nein \\
\hline
\end{tabular}

Die Online-Version des Originalartikels ist unter https://doi.org/10.1007/s11377-019-0357-y zu finden. 